\title{
Development of the 12-item questionnaire for quantitative assessment of depressive mixed state (DMX-I2)
}

This article was published in the following Dove Press journal:

Neuropsychiatric Disease and Treatment

\author{
Hotaka Shinzato' \\ Munenaga Koda' \\ Akifumi Nakamura ${ }^{1,2}$ \\ Tsuyoshi Kondo' \\ 'Department of Neuropsychiatry, \\ Graduate School of Medicine, University \\ of the Ryukyus, Okinawa, Japan; \\ ${ }^{2}$ Department of Psychiatry, Akari Clinic, \\ Okinawa, Japan
}

Background: Conventional categorical criteria have limitations in assessing the prevalence and severity of depressive mixed state (DMX). Thus, we have developed a new scale for screening and quantification of DMX and examined the symptomatological structure and severity of DMX in individuals with major depressive episode (MDE).

Methods: Subjects were 154 patients with MDE (57 males and 97 females; age 13-83 years). Our original Japanese version of the self-administered 12-item questionnaire to assess DMX (DMX-12), together with the Quick Inventory of Depressive Symptomatology SelfReport Japanese version (QIDS-SR-J) and global assessment of functioning, were administered to each participant. The symptomatological structure of the DMX-12 was examined by exploratory factor analysis. Multiple regression analyses were used to analyze factors contributing to the DMX-12 scale. The relationships of this scale with categorical diagnoses (mixed depression by Benazzi and mixed features by DSM-5) were also investigated.

Results: A three-factor model of the DMX-12 was extracted from exploratory factor analysis, namely, "spontaneous instability", "vulnerable responsiveness", and "disruptive emotion/behavior". Multiple regression analyses revealed that age was negatively correlated with total DMX-12 score, while bipolarity and the QIDS-SR-J score were positively correlated. A higher score on the disruptive emotion/behavior subscale was observed in patients with mixed depression and mixed features.

Conclusion: The DMX-12 seems to be useful for screening DMX in conjunction with conventional categorical diagnoses. Severely depressed younger subjects with potential bipolarity are more likely to develop DMX. The disruptive emotion/behavior subscale of the DMX-12 may be the most helpful in distinguishing patients with DMX from non-mixed patients.

Keywords: major depressive episode, depressive mixed state, spontaneous instability, vulnerable responsiveness, disruptive emotion/behavior

\section{Introduction}

Depressive mixed state (DMX) has been regarded as a temporary mixture of bipolar components with depressive psychopathology. ${ }^{1}$ Spontaneous verbalization of DMX symptoms is usually difficult for patients, especially when clinicians interview them mainly focusing on typical depressive symptoms, which may easily lead to the underdiagnosis of DMX. However, pharmacological treatments, mainly with antidepressants, may provoke impulsive and risky behavior, including self-harm and suicide attempts, in patients with DMX. ${ }^{2,3}$ Therefore, it is highly recommended for
Correspondence: Hotaka Shinzato Department of Neuropsychiatry, Graduate School of Medicine, University of the Ryukyus, 207 Uehara, Nishihara, Okinawa 903-0215, Japan

$\mathrm{Tel}+8|98895 \quad| 157$

Fax +8198895 1419

Email hotaka.shinzato@gmail.com 
clinicians to quickly diagnose such mixed psychopathology and respond appropriately to it as soon as possible for necessary risk management.

Kraepelin described that the mixed state is not a rare phenomenon and could be observed as various combinations of asynchronous cycles of mood, will, and thought during the course of mood disorders. ${ }^{4}$ However, the operational criteria used by the Diagnostic and Statistical Manual of Mental Disorders, 4th Edition, Text Revision $(\mathrm{DSM}-\mathrm{IV}-\mathrm{TR})^{5}$ narrowly defined a "mixed episode" as a combination of full manic and major depressive manifestations only for bipolar I disorder, which resulted in strictly limited diagnosis for mixed psychopathology during major depressive episodes (MDEs).

It was expected that the newer definition of the mixed features specifier in the Fifth Edition of DSM (DSM-5) ${ }^{6}$ would provide some beneficial effects for more sensitive and extended diagnosis of DMX. However, the prevalence of DMX still remained unexpectedly low (3.2-7.5\%) during MDEs even after the DSM-5 criteria were applied to mixed psychopathology. ${ }^{7,8}$ Several reports ${ }^{7,9-11}$ have also criticized the underdiagnosis of DMX even after using the DSM-5 criteria owing to exclusion of overlapping symptoms for both manic and depressive symptoms, such as distractibility, irritability, and psychomotor agitation during an MDE, termed a "dip" into depression by Malhi et al. ${ }^{9}$ The same authors ${ }^{9}$ have also suggested that these non-specific symptoms are rather fundamental to defining DMX. In contrast, typical hypomanic symptoms as defined for DSM-5 mixed features (ie, elevated mood, inflated self-esteem, decreased need for sleep, and increased goal-oriented activity) have been reported to be very infrequently observed during MDEs, ${ }^{8,12}$ and thus seem to be too insensitive to assess psychopathology and its severity in DMX.

Another study has also reported that aggression in MDEs is most associated with DMX. ${ }^{13}$ The definition of "mixed depression" by Benazzi, ${ }^{12,14,15}$ including these non-specific symptoms, widened the range of diagnosis for clinically relevant DMX (12.8-32.3\% even in major depressive disorder). ${ }^{8}{ }^{16}$ Thus, the prevalence of DMX has varied widely depending on its definition by various criteria. As another problem, these criteria are unlikely to be suitable for correct assessment of the severity of DMX. Consequently, these findings suggest limitations to the categorical diagnosis of DMX.

In a 2016 study, we proposed a postulated model for depressive psychopathology structured by static/dynamic and internalized/externalized dimensions, together with its plausible treatment options, and suggested the necessity of dimensional approaches to assess various types of depressive symptomatology, such as melancholic depression, psychotic depression, and mixed depression. ${ }^{17}$ It is apparent that DSM- $5^{6}$ covers only a part of DMX with combined features of MDEs and typical manic/hypomanic symptoms. By reviewing previous literature, ${ }^{7,12,18,19}$ we have concluded that the inclusion of less specific but more frequent mixed symptoms in the criteria of DMX is essential to increase the sensitivity of screening for DMX and to assess its severity. For this purpose, it seems necessary to capture the full picture of DMX on the basis of the broader definition of mixed depression by Benazzi. ${ }^{12,14,15}$ Therefore, in the present study, we developed a new scale for the screening and quantitative determination of DMX, covering non-specific but frequent mixed symptoms, and examined the symptomatological structure and severity of DMX using this scale in individuals with MDEs.

\section{Patients and methods}

\section{Subjects}

Subjects were 154 consecutively recruited patients with MDE (57 males and 97 females), who visited our clinic from June 2014 to June 2018. The mean \pm SD age was 42.6 \pm 17.4 years and the age range was from 13 to 83 years (age 13-19: $\mathrm{n}=14 ; 20-29: \mathrm{n}=24 ; 30-39: \mathrm{n}=31 ; 40-49$ : $\mathrm{n}=33 ; 50-59: \mathrm{n}=22 ; 60-69: \mathrm{n}=20 ; \geq 70: \mathrm{n}=10)$. They were currently diagnosed as having single or recurrent MDE(s) according to DSM-5 criteria by two experienced psychiatrists, and were further classified into depressive disorders $(n=111)$ and bipolar and related disorders $(n=43)$. Patients with substance-related/addictive disorders or neurocognitive disorders were excluded from analyses.

Prescribed drugs were the following: antidepressants (amitriptyline: 1; imipramine: 2; clomipramine: 1; amoxapine: 1 ; setiptiline: 1 ; trazodone: 5 ; fluvoxamine: 3 ; paroxetine: 16; sertraline: 11 ; escitalopram: 9; milnacipran: 3 ; duloxetine: 10; venlafaxine: 2; mirtazapine: 12), antipsychotic drugs (risperidone: 2 ; paliperidone: 1 ; quetiapine: 13 ; olanzapine: 5; aripiprazole: 19; sulpiride: 13; levomepromazine: 5; chlorpromazine: 1; haloperidol: 1), mood-stabilizing drugs (lithium carbonate: 10; sodium valproate: 16; lamotrigine: 10; carbamazepine: 1), benzodiazepine anxiolytics (alprazolam: 14; ethyl loflazepate: 10; lorazepam: 12; bromazepam: 7; diazepam: 3; etizolam: 26; clonazepam: 9; and clotiazepam: 3 ), benzodiazepine hypnotics (brotizolam: 21; flunitrazepam: 17; triazolam: 10; rilmazafone: 3 ; 
quazepam: 2; nitrazepam: 2; lormetazepam: 1; estazolam: 1), non-benzodiazepine hypnotics (zolpidem: 14; zopiclone: 9; eszopiclone: 9), others (methylphenidate: 1; prednisolone: 1), and no psychoactive drug: 38 .

All subjects gave written informed consent to voluntarily participate in our research. For patients under the age of 18, we obtained written informed consent not only from the patients but also from their parents. The data were anonymously treated during the study. Only coded and grouped data were used for analyses. An explanation of the purpose of the study, measures for protection of personal information, and the right to withdraw from the study were provided to each participant. This study was conducted in accordance with the Declaration of Helsinki. The study protocol was approved by the Ethics Committee of University of the Ryukyus.

\section{Assessment}

The self-administered 12-item questionnaire for assessment of depressive mixed state (DMX-12) in Japanese (Appendix 1) was developed by us (Table 1), according to previously reported core features for DMX such as distractibility, irritability, and psychomotor agitation (inner tension and restlessness) and racing/crowded thought, ${ }^{12,18}$ as well as other prevalent mixed symptoms, eg, mood lability, overreactivity, impulsivity, aggression, and risk-taking behavior, pointed out

Table I The 12-item questionnaire for assessment of depressive mixed state (DMX-I2)

\begin{tabular}{|c|c|}
\hline Evaluation item & Actual content of the questionnaire \\
\hline I. Hypersensitivity & $\begin{array}{l}\text { I am more sensitive and vulnerable than usual to } \\
\text { others' comments and attitudes }\end{array}$ \\
\hline 2. Overreactivity & $\begin{array}{l}\text { I tend to overreact to trivial things more than } \\
\text { usual }\end{array}$ \\
\hline 3. Distractibility & $\begin{array}{l}\text { I am easily distracted and unable to focus com- } \\
\text { pletely on a task }\end{array}$ \\
\hline 4. Mood lability & My mood changes rapidly in a short time \\
\hline 5. Inner tension & I feel so tense that I am unable to relax \\
\hline 6. Dysphoria & $\begin{array}{l}\text { I get overwhelmed by unpleasant and displeas- } \\
\text { ing feelings }\end{array}$ \\
\hline $\begin{array}{l}\text { 7. Racing/crowded } \\
\text { thought }\end{array}$ & $\begin{array}{l}\text { Many different thoughts run through my head } \\
\text { rapidly and fruitlessly }\end{array}$ \\
\hline 8. Restlessness & I feel restless and unable to stay still \\
\hline 9. Impulsivity & $\begin{array}{l}\text { I feel like acting impulsively with no regard for } \\
\text { consequences }\end{array}$ \\
\hline 10. Irritability & I get easily irritated for no reason \\
\hline II. Aggression & $\begin{array}{l}\text { When someone disagrees with me, I feel like } \\
\text { quarreling with or hitting that person }\end{array}$ \\
\hline $\begin{array}{l}\text { 12. Risk-taking } \\
\text { behavior }\end{array}$ & I tend to deliberately take risks \\
\hline
\end{tabular}

from previous research. ${ }^{7,19}$ This scale was translated into English (Appendix 2) and back-translated into Japanese by two native speakers of both English and Japanese, followed by confirmation from all the authors. We used the original version (in the Japanese language) in the present study. The 12 items were: hypersensitivity, overreactivity, distractibility, mood lability, inner tension, dysphoria, racing/crowded thought, restlessness, impulsivity, irritability, aggression, and risk-taking behavior (Table 1). Each item on the DMX-12 was scored using four-scale steps according to the frequency of each symptom (0: never; 1 : only occasionally; 2 : often; 3 : almost always) during the latest 1-week period of an MDE.

The DMX-12, together with the Quick Inventory of Depressive Symptomatology Self-Report ${ }^{20}$ Japanese version (QIDS-SR-J) and Global Assessment of Functioning $(\mathrm{GAF}),{ }^{21}$ were administered to each participant.

\section{Statistical analyses}

The symptomatological structure of the DMX-12 was examined in the overall patients using exploratory factor analysis after Varimax rotation. The patterns of distributions in total and subscale scores on the DMX-12 were analyzed by the Kolmogorov-Smirnov test. Effects of various factors, including age, gender, number of mood episodes, duration of illness, education level, bipolarity, severity of depression, and GAF, on total/subscale scores on the DMX-12 were examined by multiple regression analysis. Total and subscale scores on the DMX-12 were compared between patients with and without an established categorical diagnosis of DMX, defined as mixed depression ${ }^{12,14,15}$ or mixed features specifier in DSM- $5,{ }^{6}$ by the Mann-Whitney $U$-test.

A two-tailed $P$-value less than 0.05 was regarded as statistically significant. SPSS $16.0 \mathrm{~J}$ for Windows (SPSS Japan, Tokyo, Japan) was used for these statistical analyses.

\section{Results}

Exploratory factor analysis of the DMX-12 Three distinctive components of the DMX-12 were extracted from exploratory factor analysis (Table 2), namely, "spontaneous instability" (restlessness, distractibility, racing/crowded thought, mood lability, inner tension, and impulsivity: Cronbach's $\alpha=0.868$ ), "vulnerable responsiveness" (hypersensitivity and overreactivity: Cronbach's $\alpha=0.826$ ), and "disruptive emotion/behavior" (aggression, irritability, dysphoria, and risk-taking behavior: Cronbach's $\alpha=0.769$ ). 
Table 2 Exploratory factor analysis of the 12-item questionnaire for assessment of depressive mixed state (DMX-12) in 154 patients with major depressive episode

\begin{tabular}{|l|l|l|l|}
\hline \multirow{2}{*}{} & \multicolumn{3}{l}{ Factor loadings } \\
\cline { 2 - 4 } & $\mathbf{I}$ & $\mathbf{2}$ & $\mathbf{3}$ \\
\hline I. Spontaneous instability & & & \\
(Cronbach's $\alpha=0.868)$ & & & \\
Restlessness & 0.787 & 0.260 & 0.096 \\
Racing/crowded thought & 0.709 & 0.263 & 0.157 \\
Mood lability & 0.682 & 0.187 & 0.173 \\
Inner tension & 0.675 & 0.331 & 0.143 \\
Distractibility & 0.623 & 0.121 & 0.176 \\
Impulsivity & 0.547 & 0.163 & 0.293 \\
\hline 2. Vulnerable responsiveness & & & \\
(Cronbach's $\alpha=0.826)$ & & & \\
Hypersensitivity & 0.245 & 0.805 & 0.129 \\
Overreactivity & 0.368 & 0.713 & 0.279 \\
\hline 3. Disruptive emotion/beha- & & & \\
vior (Cronbach's $\alpha=0.769)$ & & & \\
Aggression & 0.050 & 0.119 & 0.873 \\
Irritability & 0.261 & 0.168 & 0.717 \\
Dysphoria & 0.380 & 0.383 & 0.449 \\
Risk-taking behavior & 0.376 & 0.117 & 0.382 \\
\hline
\end{tabular}

\section{Prevalence of DMX-I2 symptoms}

The most frequently observed persistent symptoms of the DMX-12 (>30\%) were hypersensitivity (38.3\%), racing/ crowded thought $(36.4 \%)$, distractibility (34.4\%), overreactivity (33.8\%), and inner tension $(32.5 \%)$, as shown in Figure 1. Symptoms of vulnerable responsiveness and spontaneous instability were more commonly observed than those of disruptive emotion/behavior during an MDE (Figure 1).

\section{Distribution of total and subscale scores on the DMX-12}

The distribution of total and the spontaneous instability subscale scores closely fitted the normal distribution model by the Kolmogorov-Smirnov test $(P>0.05)$, whereas a negatively skewed distribution for the vulnerable responsiveness subscale and a positively skewed distribution for the disruptive emotion/behavior subscale were found, as shown in Figure 2. The median (range) and mean \pm SD for total scores on the DMX-12 were $18(0$ 33 ) and $18.0 \pm 6.6$, respectively. The median subscale scores (range) for spontaneous instability, vulnerable responsiveness, and disruptive emotion/behavior were 10 $(0-18), 4(0-6)$, and $3(0-11)$, respectively.

\section{Multiple regression analyses of various factors affecting the DMX-12}

Multiple regression analyses (Table 3 ) revealed that age was negatively associated with total DMX-12 $(\beta=-0.300$, $P=0.001)$ and subscale scores for spontaneous instability

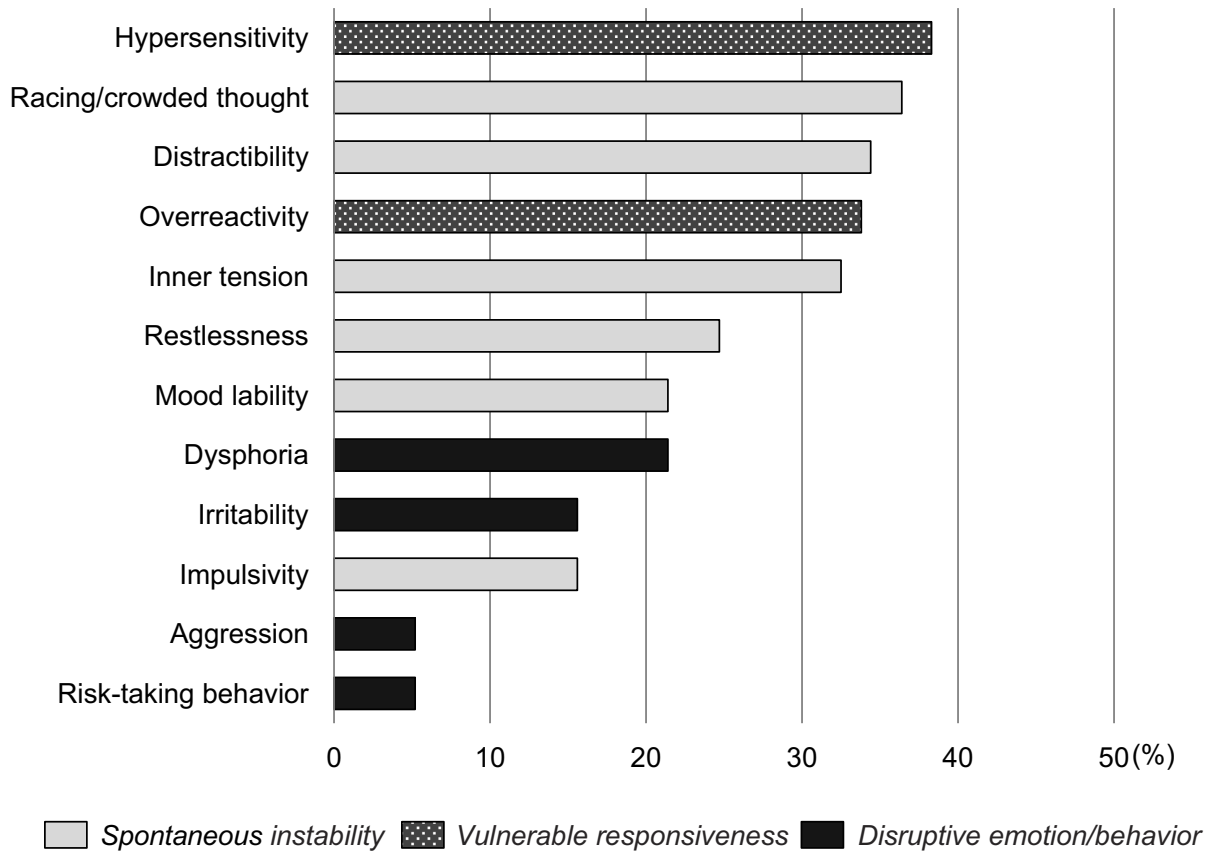

Figure I Prevalence of persistent symptoms of the 12-itemquestionnaire for assessment of depressive mixed state (DMX-I2) during a major depressive episode. 

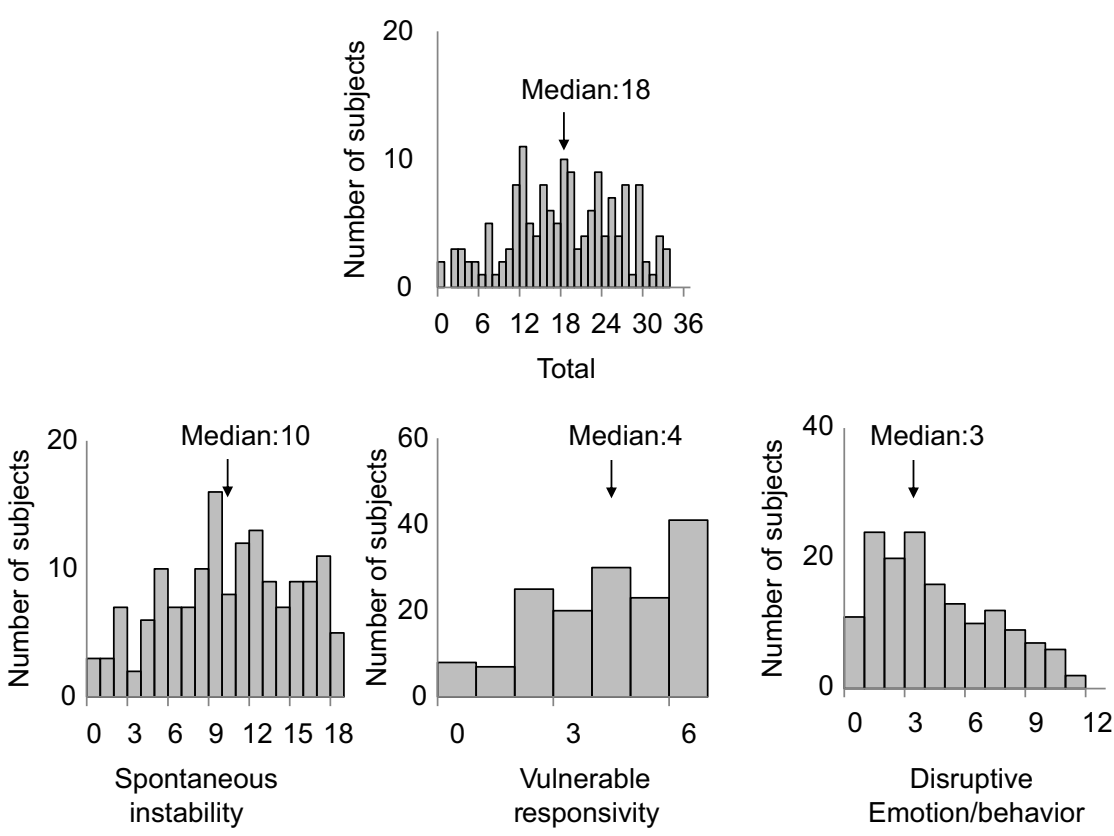

Figure 2 Histograms of total and subscale scores on the 12-item questionnaire for quantitative assessment of depressive mixed state (DMX-12). The abscissa and ordinate indicate total or subscale scores on the DMX-12 and number of subjects, respectively. Total and spontaneous instability subscale scores showed a normal distribution, whereas those for vulnerable responsiveness and disruptive emotion/behavior showed negatively and positively skewed distributions, respectively.

$(\beta=-0.192, \quad P=0.015)$ vulnerable responsiveness $(\beta=$ $-0.261, P=0.001)$, and disruptive emotion/behavior $(\beta=$ $-0.361, P=0.001)$. Meanwhile, the scores on the QIDSSR-J showed positive correlations with total scores on the DMX-12 $(\beta=0.465, P=0.001)$ and any of its subscale scores for spontaneous instability $(\beta=0.454, P=0.001)$, vulnerable responsiveness $(\beta=0.331, P=0.001)$, and disruptive emotion/behavior $(\beta=0.346, P=0.001)$. Bipolarity was positively correlated with the total DMX-12 score $(\beta=0.174, P=0.031)$ and disruptive emotion/behavior subscale score $(\beta=0.193, P=0.021)$.

\section{Categorically diagnosed DMX and the DMX-I2}

In the present study, 28 cases $(18.2 \%)$ showed mixed depression by Benazzi's criteria, ${ }^{12,14,15}$ while seven cases (4.5\%) showed a mixed features specifier during an MDE according to DSM-5 ${ }^{6}$ among the overall 154 patients with current MDEs. Comparisons of total and subscale scores on the DMX-12 were made between patients with and without categorically diagnosed DMX (Table 4). Patients with mixed depression showed higher scores than those without mixed depression on total DMX-12 (21.8 \pm 6.0 vs $17.2 \pm 8.2 ; P=0.005)$ and subscale scores of spontaneous instability $(11.7 \pm 3.9$ vs $9.7 \pm 4.9 ; \quad P=0.042)$, vulnerable responsiveness $(4.6 \pm 1.3$ vs $3.7 \pm 1.9 ; \quad P=0.027)$, and disruptive emotion/behavior $\quad(5.5 \pm 2.6 \quad$ vs $3.8 \pm 2.9$; $P=0.002)$. Meanwhile, patients with mixed features showed higher scores than those without mixed features only on the disruptive emotion/behavior subscale $(6.9 \pm 2.7$ vs $3.9 \pm 2.9 ; P=0.013$ ).

\section{Discussion}

Swann ${ }^{10}$ has implied that mixed states may be a dimensional property of mood episodes.

Challenging trials for dimensional scales have been conducted to measure mixed states, eg, the General Inventory of Mixed Affective Symptoms, ${ }^{22}$ Multidimensional Assessment of Thymic States, ${ }^{23}$ and Multiple Visual Analogue Scale of Bipolarity. ${ }^{24}$ However, these scales also include many typical symptoms with hypomania/mania and consist of 20 or more items, which may not be suitable for a compact assessment to determine the severity of DMX. The newer Koukopoulos Mixed Depression Rating Scale, proposed by Sani et al, ${ }^{25}$ should be noted as a diagnostic tool for objective and subjective assessment. However, in a busy consulting room, it may be rather time-consuming for clinicians to estimate series of depressed cases using this scale. These situations prompted us to develop the DMX-12 as a new and compact quantitative scale solely for DMX, mainly consisting of non-specific but frequently observed mixed symptoms from dimensional aspects. $^{17}$ Because the DMX-12 is a self-rating screening 

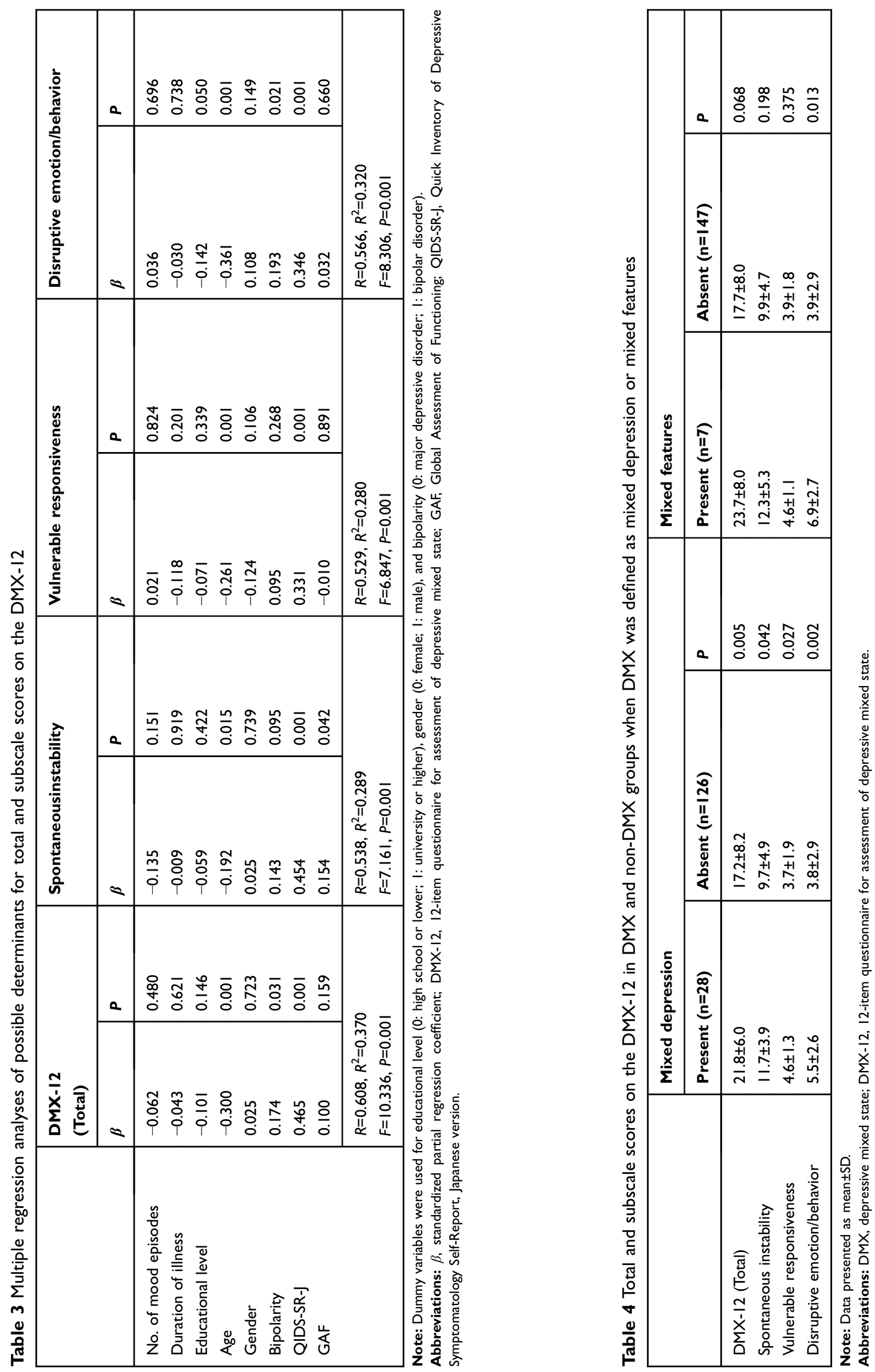
tool managed by patients themselves, it is possible to perform DMX screening easily, without inconveniencing clinicians. The DMX-12 (Table 1) almost comprehensively covers core components from the "dip" symptoms (distractibility, irritability, restlessness, and inner tension) together with other prevalent mixed symptoms such as racing/crowded thought, mood lability, overreactivity, impulsivity, aggression, and risk-taking behavior, which have been previously reported. $^{7,19}$

It should be noted that DMX symptoms were more prominent in younger depressed subjects in the present study, suggesting younger age at onset as a risk factor for mixed psychopathology (Table 3). This is consistent with previous studies, demonstrating a younger age with temperamental mood lability ${ }^{26}$ and an earlier age at onset of the illness ${ }^{27,28}$ in DMX patients. These may at least partly support an association between age and DMX in the context of temperamental vulnerability and potential bipolarity.

Previous reports have also suggested a direct relationship between bipolarity and DMX. ${ }^{8,12,27,28}$ In fact, bipolarity had positive effects on the total DMX-12 and disruptive emotion/behavior subscale scores in the present study. These results suggest the necessity to recognize potential bipolarity in depressed individuals manifested by disruptive emotion/behavior symptoms, ie, dysphoria, irritability, aggression, and risk-taking behavior, even though they do not apparently fulfill criteria for hypomanic psychopathology. Thus, the presence of the disruptive emotion/behavior subscale symptoms during an MDE may be indicative of the future need for bipolar treatments.

Meanwhile, it is obvious that the severity of DMX is strongly dependent on that of core depressive symptoms (Table 3). This may imply that the presence of clinically significant DMX is regarded as an expression of severe depression, which may need more intensive treatment intervention than non-mixed depression. Considering the fact that the intrusion of mixed components into depression not only increases the severity of depression but also requires more potent medication, ie, a shift from antidepressants to mood-stabilizing agents including atypical antipsychotics, ${ }^{29}$ the biological basis of DMX in the context of severity of depression needs to be clarified in the future.

In the present study, exploratory factor analysis revealed a three-factor model for the symptomatological structure of the DMX-12, consisting of the spontaneous instability, vulnerable responsiveness, and disruptive emotion/behavior subscales (Table 2). Patterns of distributions were apparently different among the scores on these three subscales in the overall patients with MDEs, ie, spontaneous instability was normally distributed, vulnerable responsiveness was negatively skewed, and disruptive emotion/behavior was positively skewed (Figure 2), which may indirectly support the three-factor model as independent components.

A considerable number of patients with MDEs have various degrees of spontaneous instability symptoms according to the normal distribution (Figures 1 and 2). The spontaneous instability subscale contains instability in thought process (racing/crowded thought and distractibility), mood state (inner tension, restlessness, and mood lability), and behavioral tendency (impulsivity). It is assumed that these spontaneous instability symptoms impair patients' capability to maintain positive attention, good concentration, rational decision-making, and thoughtful behavior, thereby leading to a readiness for careless, inconsistent, and impulsive responses to reality during depressive mixed episodes.

The vulnerable responsiveness symptoms, such as hypersensitivity and overreactivity, are common in most patients with MDEs (Figures 1 and 2). Despite frequently observed vulnerable responsiveness symptoms, such internalized features are not verbally expressed by patients with MDEs and are unlikely to be focused on or intensively interviewed as an extended depressive psychopathology by clinicians. However, it is possible that hypersensitivity and overreactivity to stimuli from surrounding others and situations, together with lowered tolerance to inner frustration and anxiety, may easily provoke loss of cognitive control and mood dysregulation in patients with DMX during MDEs. Therefore, an assessment of the vulnerable responsiveness component appears to be important for clinicians to plan a more deliberate strategy for cognitive behavioral therapy to enhance patients' self-awareness and self-control, and to avoid self-induced deterioration in mood symptoms.

The disruptive emotion/behavior symptoms, featuring dysphoria, irritability, aggression, and risk-taking behavior, are much less frequently observed during MDEs than any other DMX-12 subscales (Figures 1 and 2), but are possibly helpful in distinguishing patients with DMX from those without DMX, by both Benazzi's definition ${ }^{12,14,15}$ and DMX $-5^{6}$ criteria (Table 4$)$. These symptoms have also been regarded as promoting factors for suicidal behavior, ${ }^{7,30,31}$ to which clinicians should pay the greatest attention in 
assessing and managing the suicidal risks of patients with DMX. Presumably, the disruptive emotion/behavior symptoms may be essential and core characteristics of DMX even when different categorical criteria are applied., 7,16 Furthermore, a report suggesting aggressiveness in depression as a feature of $\mathrm{DMX}^{13}$ may also support the findings on disruptive behavior in the present study. The disruptive emotion/behavior symptoms are relatively apparent to surrounding others and are sometimes externalized as interpersonal friction and dangerous acting out. Accordingly, clinicians should carefully confirm whether such symptoms have intruded only during mood episodes, and may need to conduct differential diagnosis of DMX during MDEs from borderline personality disorder or disruptive, impulse control, and conduct disorders, which rather constantly involve interpersonal or behavioral problems irrespective of episodic mood alterations.

As for the total score on the DMX-12, the median score was 18 (36 as the full mark) in the overall patients (Figure 2). This indicates that DMX ranging from mild to severe degree during MDEs seems to be a commonly observed phenomenon in a real-world clinical setting. Before starting our study, it was hypothesized that patients with DMX would show more severe disturbance in their daily and social life than those without DMX. Contrary to our clinical impression, the overall severity of mixed symptoms assessed by total DMX-12 score was not directly associated with patients' GAF (Table 3). Despite this negative result, clinicians should be warned that DMX symptoms often provoke risky behavior, including suicide attempts. $^{7,30,31}$

This is a preliminary study and has several limitations. First, the results were obtained from a relatively small number of Japanese subjects. Second, since the DMX-12 included many non-specific symptoms, its specificity for the diagnosis of DMX was not sufficiently established. Third, assessment using frequency rather than severity of DMX was conducted on the basis of self-report scales by the DMX-12. Fourth, the reliability and validity of the English version of the DMX-12 have not yet been justified in other ethnic groups. Therefore, the factor model of the DMX-12 should be re-examined in larger number of subjects with various ethnicity and languages. The specificity of the DMX-12 for DMX may also be confirmed not only in depressed patients but also in non-depressed subjects, eg, those with anxiety disorders and neurodevelopmental disorders. Confirmatory analyses may be required in the future, using new valid methods such as clinimetric approaches, as suggested by Carrozzino et al. ${ }^{32}$

Nevertheless, the quantification of DMX using the DMX-12 may open the door not only to routine screening for DMX with considerable severity but also to assessments of treatment response to probe drugs for DMX through visible intra-individual changes in mixed psychopathology.

\section{Conclusions}

The DMX-12 seems to be easy to use and helpful in screening for DMX, in good agreement with conventional categorical diagnoses. The DMX-12 has revealed that various degrees of DMX during MDEs are commonly observed in a real-world clinical setting. Clinicians should be more sensitive to DMX, especially in severely depressed younger individuals with potential bipolarity. The disruptive emotion/behavior subscale of the DMX12 may be the most helpful in distinguishing patients with DMX from those without mixed psychopathology.

\section{Acknowledgments}

This study was supported by JSPS KAKENHI (grant number JP17K10311). The funders had no role in study design, data collection, analysis, writing of the manuscript, or decision to publish.

\section{Disclosure}

Dr Hotaka Shinzato reports grants from JSPS KAKENHI, during the conduct of the study. Dr Akifumi Nakamura has received honoraria from Meiji Seika Pharma and Otsuka. The authors report no other conflicts of interest in this work.

\section{References}

1. Fornaro M, Martino M, De Pasquale C, Moussaoui D. The argument of antidepressant drugs in the treatment of bipolar depression: mixed evidence or mixed states? Expert Opin Pharmacother. 2012;13:20372051. doi:10.1517/14656566.2012.719877

2. Koukopoulos A, Sani G, Koukopoulos AE, Manfredi G, Pacchiarotti I, Girardi P. Melancholia agitata and mixed depression. Acta Psychiatr Scand. 2007;115(Suppl. 433):50-57. doi:10.1111/j.1600-0447.2 007.00963.x

3. Swann AC. Activated depression: mixed bipolar disorder or agitated unipolar depression. Curr Psychiatry Rep. 2013;15:376. doi:10.1007/ s11920-013-0376-1

4. Kraepelin E. Psychiatrie: EinLehrbuch Für Studierende Und Ärzte, Achte Auflage. Leipzig: Johann Ambrosius Barth; 1913.

5. American Psychiatric Association. Diagnostic and Statistical Manual of Mental Disorders. 4th edn, text version ed. Washington, DC: American Psychiatric Association; 2000. 
6. American Psychiatric Association. Diagnostic and Statistical Manual of Mental Disorders. 5th ed. Washington, DC: American Psychiatric Association; 2013.

7. Perugi G, Angst J, Azorin J-M, et al. BRIDGE-II-mix study group. Mixed features in patients with a major depressive episode: the BRIDGE-II-Mix study. J Clin Psychiatry. 2015;76:e351-e358. doi:10.4088/JCP.14m09092

8. Takeshima M, Oka T. DSM-5-defined 'mixed features' and benazzi's mixed depression: which is practically useful to discriminate bipolar disorder from unipolar depression in patients with depression? Psychiatry Clin Neurosci. 2015;69:109-116. doi:10.1111/pcn.12213

9. Malhi GS, Fritz K, Allwang C, et al. Are manic symptoms that "dip" into depression the essence of mixed features? J Affect Disord. 2016;192:104-108. doi:10.1016/j.jad.2015.12.009

10. Swan AC. Mixed features: evolution of the concept, past and current definitions, and future prospects. CNS Spectr. 2017;22:161-169. doi:10.1017/S1092852916000882

11. Ogasawara K, Nakamura Y, Kimura H, Ozaki N. Issues on the diagnosis and etiopathogenesis of mood disorders: reconsidering DSM-5. J Neural Transm. 2018;125:211-222. doi:10.1007/s00702-017-1828-2

12. Benazzi F. Defining mixed depression. Prog Neuro-Psychopharmacol Biol Psychiatry. 2008;32:932-939. doi:10.1016/j.pnpbp.2007.12.019

13. Verdolini N, Perugi G, Samalin L, et al. Aggressiveness in depression: a neglected symptom possibly associated with bipolarity and mixed features. Acta Psychiatr Scand. 2017;136(4):362-372. doi:10.1111/acps. 12777

14. Benazzi F. Which could be a clinically useful definition of depressive mixed state? Prog Neuro-Psychopharmacol Biol Psychiatry 2002; 26:1105-1111

15. Benazzi F. Bipolar disorder - focus on bipolar II disorder and mixed depression. Lancet. 2007;369:935-949. doi:10.1016/S0140-6736(07) 60453-X

16. Benazzi F. A tetrachoric factor analysis validation of mixed depression. Prog Neuro-Psychopharmacol Biol Psychiatry. 2008;32:186192. doi:10.1016/j.pnpbp.2007.08.005

17. Kondo T, Shinzato H, Koda M. Diagnostic and therapeutic considerations in depressive mixed state. Clin Neuropsychopharmacol Ther 2016;7:41-47. doi:10.5234/cnpt.7.41

18. Malhi GS, Lampe L, Coulson CM, et al. Mixed state discrimination: a DSM problem that won't go away? J Affect Disord. 2014;158:8-10. doi:10.1016/j.jad.2014.01.008

19. Sani G, Vohringer PA, Napoletano F, et al. Koukopoulos's diagnostic criteria for mixed depression: a validation study. J Affect Disord. 2014;164:14-18. doi:10.1016/j.jad.2014.03.054

20. Rush AJ, Trivedi MH, Ibrahim HM, et al. The 16-item Quick Inventory Of Depressive Symptomatology (QIDS), clinician rating (QIDS-C), and selfreport (QIDS-SR): a psychometric evaluation in patients with chronic major depression. Biol Psychiatry. 2003;54:573-583.
21. Endicott J, Spitzer RL, Fleiss JL, Cohen J. The global assessment scale: a procedure for measuring overall severity of psychiatric disturbance. Arch Gen Psychiatry. 1976;33:776-779.

22. Bertschy G, Gervasoni N, Favre S, et al. Phenomenology of mixed states: a principal component analysis study. Bipolar Disord. 2007;9:907-912. doi:10.1111/j.1399-5618.2007.00462.x

23. Henry C, M'Bailara K, Mathieu F, Poinsot R, Falissard B. Construction and validation of a dimensional scale exploring mood disorders: mAThyS (multidimensional assessment of thymic states). BMC Psychiatry. 2008;8:82. doi:10.1186/1471-244X-8-82

24. Azorin JM, Kaladjian A, Adida M, et al. Self-assessment and characteristics of mixed depression in the French national EPIDEP study. $J$ Affect Disord. 2012;143:109-117. doi:10.1016/j.jad.2012.05.036

25. Sani G, Vöhringer PA, Barroilhet SA, Koukopoulos AE, Ghaemi SN. The Koukopoulos Mixed Depression Rating Scale (KMDRS): an International Mood Network (IMN) validation study of a new mixed mood rating scale. J Affect Disord. 2018;232:9-16. doi:10.1016/j.jad.2018.01.025

26. Benazzi F. Impact of temperamental mood lability on depressive mixed state. Psychopathology. 2006;39:19-24. doi:10.1159/ 000089659

27. Takeshima M, Oka T. A comprehensive analysis of features that suggest bipolarity in patients with a major depressive episode: what is the best combination to predict soft bipolarity diagnosis? J Affect Disord. 2013;147:150-155. doi:10.1016/j.jad.2012.10.026

28. Inoue T, Inagaki Y, Kimura T, Shirakawa O. Prevalence and predictors of bipolar disorders in patients with a major depressive episode: the Japanese epidemiological trial with latest measure of bipolar disorder (JET-LMBP). J Affect Disord. 2015;174:535-541. doi:10.1016/j.jad.2014.12.023

29. Grunze H, Vieta E, Goodwin GM, et al. Members of the WFSBP task force on bipolar affective disorders working on this topic. The World Federation of Societies of Biological Psychiatry (WFSBP) guidelines for the biological treatment of bipolar disorders: acute and long-term treatment of mixed states in bipolar disorder. World $J$ Biol Psychiatry. 2018;19:2-58. doi:10.1080/15622975.2017. 1384850

30. Popovic D, Vieta E, Azorin JM, et al. Suicide attempts in major depressive episode: evidence from the BRIDGE-II-mix study. Bipolar Disord. 2015;17:795-803. doi:10.1111/bdi.12338

31. Persons JE, Coryell WH, Solomon DA, Keller MB, Endicott J, Fiedorowicz JG. Mixed state and suicide: is the effect of mixed state on suicidal behavior more than the sum of its parts? Bipolar Disord. 2018;20:35-41. doi:10.1111/bdi.12538

32. Carrozzino D, Svicher A, Patierno C, Berrocal C, Cosci F. The euthymia scale: a clinimetric analysis. Psychother Psychosom. 2019;88(2):119-121. doi:10.1159/000496230
Neuropsychiatric Disease and Treatment

\section{Publish your work in this journal}

Neuropsychiatric Disease and Treatment is an international, peerreviewed journal of clinical therapeutics and pharmacology focusing on concise rapid reporting of clinical or pre-clinical studies on a range of neuropsychiatric and neurological disorders. This journal is indexed on PubMed Central, the 'PsycINFO' database and CAS, and is the official journal of The International Neuropsychiatric Association (INA). The manuscript management system is completely online and includes a very quick and fair peer-review system, which is all easy to use. Visit http://www.dovepress.com/testimonials.php to read real quotes from published authors. 